Questions vives

\section{Questions Vives}

Recherches en éducation

$N^{\circ} 28 \mid 2017$

De l'indifférenciation à la différenciation

\title{
Cassirer, la critique de la confusion dans la culture comme transvaluation mythico-magique
}

Cassirer, the analysis of confusion in culture as mythico-magic transvaluation

\section{Leonore Bazinek et Marie-Louise Martinez}

\section{(2) OpenEdition}

1 Journals

\section{Édition électronique}

URL : http://journals.openedition.org/questionsvives/2754

DOI : 10.4000 /questionsvives. 2754

ISSN : 1775-433X

Éditeur

Université Aix-Marseille (AMU)

\section{Édition imprimée}

Date de publication : 29 décembre 2017

ISBN : 978-2-912643-52-0

ISSN : $1635-4079$

\section{Référence électronique}

Leonore Bazinek et Marie-Louise Martinez, « Cassirer, la critique de la confusion dans la culture comme transvaluation mythico-magique », Questions Vives [En ligne], $N^{\circ} 28$ | 2017, mis en ligne le 15 novembre 2018, consulté le 10 décembre 2020. URL : http://journals.openedition.org/questionsvives/ 2754 ; DOI : https://doi.org/10.4000/questionsvives.2754

Ce document a été généré automatiquement le 10 décembre 2020.

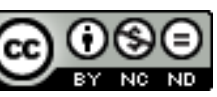

Questions Vives est mis à disposition selon les termes de la licence Creative Commons Attribution -

Pas d'Utilisation Commerciale - Pas de Modification 4.0 International. 


\section{Cassirer, la critique de la confusion dans la culture comme transvaluation mythico-magique}

Cassirer, the analysis of confusion in culture as mythico-magic transvaluation

Leonore Bazinek et Marie-Louise Martinez

\section{Introduction}

1 Aujourd'hui se manifestent des formes culturelles hybrides inquiétantes qui demandent à être réinterrogées. En effet, l'ordre et la logique de l'échange économique comme marchandisation se rabattent sur la diversité des formes de l'échange symbolique jusqu'au cœur des institutions de la connaissance (l'école ou l'université). De même, dans divers pays, la distance entre le religieux et le mythico-magique sacrificiel s'amenuise et vient submerger le politique et le juridique. La crise des institutions culturelles, dans un contexte d'indifférenciation aggravé par la globalisation, encourage ces confusions catégorielles. Des catégories du sacré archaïque, comme le bouc émissaire, surgissent jusque dans les démocraties qui prétendent s'y opposer.

2 Devant ces perspectives, il est opportun de revenir à la réflexion d'Ernst Cassirer (1874-1945) sur une philosophie de la culture. Cassirer a montré comment l'homme se développe à travers les diverses formes symboliques qu'il a lui-même engendrées et qui composent la culture comme une véritable « unité systématique ( (Cassirer, 1972, p. 8). Ses travaux permettent d'étudier certaines manifestations passées ou actuelles de crise et de confusion dans la culture, qu'il appréhende comme «transvaluation de toutes nos valeurs » (Cassirer 1993, p. 382).

3 À travers la philosophie des formes symboliques, on peut comprendre la culture comme genèse et ensemble organisé, mais aussi saisir comment le désordre et le chaos s'instaurent et sèment la confusion entre les domaines. Cassirer attire notre attention sur l'intérêt de la clarification de la séparation des ordres et des catégories symboliques dans 
la rationalité et, au contraire, sur les risques de leur indistinction liée au mythique. C'est en repérant la place singulière accordée au mythe et au rite, avec leurs puissances, qu'il devient possible de poser les bases d'une critique de la culture.

4 Très tôt, dès 1923 [1925 ?], le philosophe a repéré la place fondatrice du rite et du mythe à l'origine de la genèse culturelle, à l'instar de certains anthropologues qu'il fréquente assidûment dans le souci de suivre de près les avancées des sciences sociales. Comme le Durkheim des Fondements élémentaires de la vie religieuse (1912) qu'il connaît bien, Cassirer voit dans la forme mythique l'origine des autres systèmes symboliques. Toutes les formes symboliques, notamment l'art, le religieux, le scientifique, naissent au sein du mythicomagique pour s'en détacher progressivement par un processus continu de sécularisation.

Cassirer (1993) fait partie des penseurs qui ont su voir la violence de l'indifférenciation dans la culture, comme transgression des ordres et catégories, en lien avec le mythe. Comme l'avait en partie saisi Durkheim (1998), quarante-trois ans avant lui, dans La division du travail social et comme Girard (1972) l'expliquera magistralement, vingt-six ans après lui, dans La violence et le sacré, l'indifférenciation confusionnelle secrète vite le retour mythique à l'expulsion du bouc émissaire suivi par une redifférenciation ségrégative et violente du social. Comme ces penseurs de l'anthropologie, Cassirer (1972) accorde une place singulière à la catégorie du bouc émissaire, qu'il explique par les descriptions ethnographiques comme rite de purification et comme acte de «transmission réelle et physique » (p. 80). Il a cherché, lui aussi, à comprendre les processus avec leurs effets, il s'est demandé comment les cultures avaient pu céder ou résister à ces forces obscures, particulièrement lors du reflux mythique avec la prédominance du mécanisme d'éviction du bouc émissaire. Girard (2004), dans Les origines de la culture, ira jusqu'à accorder un pouvoir morphogénétique et anthropogénétique aux processus du bouc émissaire. Cassirer pensera jusqu'à son dernier souffle aux conditions des métamorphoses du mythico-magique vers ses issues catastrophiques ou au contraire vers ses mutations édifiantes : éthico-religieuses ou éthico-politiques.

6 L'intérêt de l'œuvre de Cassirer, exceptionnelle chez les philosophes, est d'avoir su décrire et saisir la culture en prise avec les puissances de la confusion mythico-magique, particulièrement lors de la crise du nazisme. Dans cet article, nous nous appuyons sur le potentiel critique de deux ouvrages majeurs, La pensée mythique, publié en 1925 à Hambourg, et Le mythe de l'État, écrit à la veille de sa mort, en avril 1945, et édité par deux collègues de Yale en 1946. Vingt années de maturation séparent ces deux livres, dont treize années d'exil passées à considérer l'hitlérisme et ses effets de distorsions idéologiques violentes sur la culture.

7 Nous présentons les grandes lignes de l'analyse du mythique tracées dans le volume deux de La philosophie des formes symboliques (Cassirer, 1972 [1925]), mais aussi celles du retour catastrophique et manipulé du mythique dans Le mythe de l'État (Cassirer, 1993 [1946]). Si l'auteur a vu d'abord la puissance d'engendrement par le mythe des formes les plus hautes de la culture, plus tard, poussé par son expérience et la demande de ses proches, en scrutant l'hitlérisme, il a su découvrir les violences d'un redéploiement du mythicomagique, mutilant la culture. 


\section{Le mythe : une position première et originelle de la conscience humaine}

Inspiré par la critique faite à Descartes par Leibniz (Cassirer, 1962) selon laquelle il faudrait faire une plus grande place à l'expérience, Cassirer se tourne vers les sciences sociales et les sciences du langage naissantes, il dépeint donc l'homme comme " animal symbolique " et la culture comme un ensemble de "systèmes symboliques ». Comme l'affirme Birgit Recki (2013) de l'université de Hambourg, qui a dirigé l'édition des đuvres complètes de Cassirer en vingt-cinq volumes (Hamburger Ausgabe, 1998-2007), Cassirer a eu l'ambition d'élargir les concepts aristotéliciens d'animal politique ou d'animal rationnel en forgeant celui d'animal symbolique. Il « comprend l'homme comme cet être qui apporte au monde la signification : l'être qui produit et qui comprend les symboles", le concept de la culture «signifie ici la forme de vie de l'homme, la réalité produite par l'homme comme univers de signification ", « la culture est le système vivant des formes symboliques ayant chacune sa propre dynamique et détermination par ses principes et médias propres » (pp. 113-115). Pour Cassirer, le mythe n'est pas seulement le grand récit des origines, mais une position intemporelle de la conscience dominée par des émotions, par l'absence de distance réflexive dans l'usage des images ou d'autres symboles. Le mythe est le sol nourricier de la culture, la forme symbolique fondatrice et archaïque à partir de laquelle les autres formes symboliques se développent par différenciation et sécularisation. Il importe alors de comprendre comment le mythe ou mythico-magique accompagne l'évolution de la pensée et de la culture.

\section{Le mythe : sa place et sa fonction dans les systèmes symboliques}

9 Pour Cassirer (1972), le développement de l'humain, prise de conscience de l'esprit autant que genèse de la culture, a été possible grâce aux formes symboliques engendrées et leur articulation en « unité systématique » dépassant largement l'état d'« un simple agrégat ». «Unité organique de l'esprit » (p. 8) dans laquelle la pensée mythique :

occupe [...] une place décisive : cela apparaît immédiatement quand on considère la genèse des formes fondamentales de la culture spirituelle qui toutes ont leur origine dans la conscience mythique. Aucune de ces formes ne possède initialement un être autonome et une figure particulière clairement définie. Elles apparaissent toutes au contraire comme vêtues et enveloppées dans quelque figure issue du mythe. Il n'y a guère un domaine de l'esprit objectif dont on ne puisse montrer qu'il a formé, à l'origine, avec le mythe une unité concrète et intégrée de ce genre. Les produits de l'art, comme ceux de la connaissance, les contenus de la morale, du droit, du langage, de la technique, nous renvoient à cette relation fondamentale (pp. 8-9).

Après avoir tout spécialement souligné la fonction du langage, l'auteur se prononce sur la genèse de la culture dans le mythe :

Les notions théoriques fondamentales de la connaissance, les notions d'espace, de temps, de nombre, les notions juridiques et sociales, comme la notion de propriété, ainsi que d'ailleurs chacune des constructions particulières de l'économie, de l'art, et de la technique, ne se libèrent que très progressivement de cet environnement et de ces attaches mythiques (p. 9). 

Elle est fondée sur une pratique des rites qui entraîne participation et transsubstantiation. Par l'exercice de ces rites, l'homme s'empare de son pouvoir sur la nature, si bien qu'il devient lui-même le « dieu » auquel est destiné le rituel et qu'il est en train de représenter (pp.60-61). La conscience mythique ne peut alors pas distinguer entre les choses et les signes, ce qui explique la croyance en une prise immédiate des mots sur la réalité. « L'hypothèse décisive est que le mot et le nom n'ont pas simplement une fonction de représentation et qu'ils renferment au contraire l'objet lui-même avec ses forces réelles ». Voilà la condition de possibilité des pratiques magiques: pour la pensée mythique, les mots agissent vraiment. «Le nom et la personnalité se confondent ici » (pp. 62-63).

11 Ainsi, on fait subir à l'image d'une personne un sortilège, et elle est censée être atteinte (pp. 64-65). Il y a bien une conception mythique de proto-causalité, différente de ce que deviendra la causalité véritable dans les sciences. Quand l'esprit mythique s'attache aux explications individuelles, l'esprit scientifique généralise :

Tout se passe comme si la conscience cognitive pure et la conscience mythique plaçaient le levier de l'explication à des endroits tout à fait différents. La première est satisfaite quand elle parvient à saisir l'événement individuel dans l'espace et le temps comme cas d'espèce d'une loi universelle, tandis qu'elle ne s'interroge pas plus avant sur l'individualisation, le " pourquoi » de l'ici et du maintenant en tant que tel. La seconde au contraire dirige précisément cette question du " pourquoi » sur le particulier, le singulier et l'instant unique. Elle "explique " l'événement individuel en posant et en admettant des actes volontaires individuels (p. 71).

La substantialisation et la transsubstantiation sont des processus du mythico-magique, constituant une véritable ontologisation: la qualité matérielle attachée à une chose devient réelle et peut être transmise à une autre. Ainsi, l'alchimie repose sur la pensée mythique, car l'examen des procédés alchimiques montre qu'ils «se fondent sur l'idée essentielle qu'on peut transmettre et matériellement détacher, comme une chose, les qualités et les états" (p.92). Cassirer veut cependant insister sur les propriétés ambivalentes du mythique qui peuvent permettre aussi des avancées civilisationnelles.

\section{Les mutations internes du mythico-magique à l'éthico-religieux}

À l'intérieur même de la conscience mythico-magique se prépare déjà l'éthico-religieux, sursaut bien distinct, pour Cassirer. Ce pas s'effectuerait au moment où l'homme reconnaît qu'il n'est pas seulement dirigé par une force obscure, susceptible de lui nuire, mais qu'il peut y avoir un esprit protecteur. Cette prise de conscience des deux visages du sacré, l'un numineux et l'autre terrifiant, se développe dans la relation que l'homme entretient avec lui-même. Cette expérience le rend lentement capable de développer une relation de confiance dès lors qu'il ne la ressent plus comme menaçante. «Lorsque l'homme s'élève de la magie à la religion et cesse de craindre les démons pour croire en certains dieux et leur rendre un culte, cette apothéose est moins tournée vers l'extérieur que l'intérieur " (Cassirer, 1972, pp. 199-200). Cette mutation va permettre la naissance individuelle à la manière de

la mutation sémantique que subissent en latin et en grec les termes qui désignent le daîmon et le genius [...] le génie comme son nom l'indique est celui qui « engendre » 
véritablement l'homme, et non seulement au sens physique mais au sens spirituel :

c'est l'origine et l'expression de sa personnalité (p. 201). mécanisme doit être dénoncé comme une régression dans un état culturel avancé. Il voit dans le nazisme, dont il a pu mesurer le coût humain et culturel, une instrumentalisation politique du bouc émissaire à grande échelle. À la demande de ses amis et collègues américains, Cassirer a rédigé un texte pour tenter de comprendre l'hitlérisme. Le philosophe, contre ses habitudes, a accepté de le publier dans une revue grand public (Flood, éd., 2008), il l'a retravaillé ensuite pour en faire un livre consistant, Le mythe de l'État (Cassirer, 1993), son dernier ouvrage, ressource pour élaborer une critique des cultures.

\section{Le redéploiement du mythico-magique et de ses confusions au service d'une technique de fabrication des mythes}

17 La philosophie des formes symboliques de Cassirer a contribué à cerner la place ambivalente du mythe et du rite. Le mythique travaille à la genèse de la culture et de la civilisation, mais son retour en furie défigure les cultures. Celui-ci a été repéré par Cassirer dans l'Allemagne nazie, comme dans le contexte des grands totalitarismes de la première moitié du $\mathrm{XX}^{\mathrm{e}}$ siècle. Nous pourrions en trouver les caractéristiques dans la grande crise actuelle, occasionnée par la mondialisation néolibérale. Entre 1925, où il publie le second volume de la Philosophie des formes symboliques, et 1944, où il rédige son dernier livre, le philosophe a vu un monde bouleversé de fond en comble. Comment la régression barbare a-t-elle été possible ? Cassirer (1966) tente d'y apporter explication en accord avec La philosophie des Lumières, à laquelle il a consacré un ouvrage en 1932, et dont il reste un fidèle admirateur. La découverte du logos, l'engendrement de la rationalité, ont été décisifs pour que l'homme puisse procéder à l'organisation de la vie et s'affranchir des forces obscures du mythos. L'auteur maintiendra cette position rationaliste malgré les 
voix très critiques qui ont déjà commencé à se faire entendre à son époque et qui vont encore s'amplifier dans les décennies ultérieures. Il admet cependant que la découverte de la rationalité n'a pas déclenché un processus linéaire du progrès : «En politique, on vit sans cesse sur une terre volcanique. Il faut toujours se tenir prêt à faire face à des convulsions ainsi qu'à des éruptions. À chaque moment critique de la vie sociale». (Cassirer, 1993, p. 378). Pour lui, la latence du mythico-magique peut toujours surgir de façon catastrophique selon les circonstances. C'est le cas du nazisme, quand le contexte économico-politico-social se prêtait à un retour farouche de l'irrationnel, sorte d'ultima ratio brutal du mythico-magique, comme l'explique le philosophe :

en un temps d'inflation et de chômage, l'ensemble du système économique et social de l'Allemagne était menacé de sombrer dans un effondrement complet. Toutes les ressources étaient apparemment épuisées. C'est sur ces germes que les mythes politiques ont pu croître et c'est là qu'ils ont pu trouver leur aliment. [...] Dans les situations désespérées, l'homme a toujours recours à des moyens désespérés et nos mythes politiques contemporains sont de tels moyens désespérés. Quand la raison nous manque, il reste toujours l'ultima ratio (pp. 375, 377).

L'auteur ira plus loin dans la vision d'une fabrication rationnelle et instrumentalisée de l'irrationalité. Dans la troisième partie, «Le mythe du XXe siècle » (pp. 257-402), dans le chapitre XVIII, « La technique des mythes politiques modernes » (pp. 374-400), il suggère que la diffusion de tels mythes politiques, loin d'être «le résultat d'une activité inconsciente et libre produit de l'imagination» est le résultat d'une fabrication méthodique :

Les politiciens modernes [...] ont dû agir simultanément comme homo magus et homo faber. Ils sont devenus les prêtres d'une nouvelle religion entièrement irrationnelle et mystérieuse, tout en procédant très méthodiquement, dès qu'il s'agissait de défendre et de propager cette religion. Rien n'a été laissé au hasard; toute démarche a été soigneusement préparée et préméditée. C'est cette étrange combinaison qui est l'un des traits les plus marquants de nos mythes politiques modernes. [...] Les nouveaux mythes politiques [...] sont des choses artificielles, fabriquées par des artisans habiles et rusés. Il a été réservé au $\mathrm{XX}^{\mathrm{e}}$ siècle, notre grand siècle technique, de développer une nouvelle technique du mythe. Désormais les mythes peuvent être manufacturés au sens et selon les mêmes méthodes où le sont n'importe quelles autres armes-mitrailleuses ou avions. C'est quelque chose de nouveau - quelque chose d'une importance cruciale. La forme entière de notre vie sociale en a été transformée (pp. 380-381).

Voilà, selon le philosophe, comment la confusion mythico-magique a été instrumentalisée dans le creuset du technico-politique et de la propagande pour donner lieu à un véritable « réarmement mental»:

C'est en 1933 que le monde politique commença à déplorer plus ou moins le réarmement de l'Allemagne et ses possibles répercussions internationales. En réalité, ce réarmement avait commencé bien des années plus tôt, mais il était passé presqu'inaperçu. Ce réarmement réel a commencé avec la naissance et la montée des mythes politiques. Le réarmement militaire ultérieur n'en a été que la conséquence accessoire. Ce fait était un fait accompli bien avant; le réarmement militaire ne fut que la conséquence nécessaire du réarmement mental réalisé par les mythes politiques (p. 381).

C'est à travers la manipulation du langage, dans sa fonction magique et performative de propagande que le premier pas est franchi :

Le magicien ou sorcier est seul capable de gouverner la parole magique. Mais elle devient dans ses mains la plus puissante des armes. Il est assez curieux que tout cela réapparaisse dans notre monde moderne. Quand on étudie les mythes politiques 
modernes et l'usage qu'on en fait, on trouve à notre grande surprise, non seulement une transvaluation de toutes nos valeurs éthiques, mais également une transformation du langage humain. Le mot magique l'emporte sur le mot sémantique. [...] des mots qui étaient utilisés auparavant dans un sens descriptif, logique ou sémantique, le sont maintenant d'une façon magique, destinée à produire certains effets [...] chargés d'affects et de passions violentes (pp. 381-382).

L'auteur découvre, avec stupeur, la force inquiétante des nouveaux termes produits par le régime nazi. Il suffit d'écouter ces nouveaux mots pour découvrir en eux tout un mélange d'émotions humaines - de haine, de colère, de fureur, de morgue, de mépris, d'arrogance et de dédain (pp.382-383). De telles métamorphoses avec distorsions ne sont pas totalement inédites, elles ont très certainement déjà existé dans l'histoire, donnant lieu à d'autres mutations culturelles. Cassirer, avant le totalitarisme nazi, en a étudié les manifestations dans la crise du dionysisme en Grèce antique de la fin du VI ${ }^{\mathrm{e}}$ siècle avant notre ère. Crise du sens des valeurs, violences religieuses qui furent surmontées bien plus tard dans la mutation vers une spiritualité éthico-religieuse ou éthico-politique. Il en voit les traces dans la tragédie d'Euripide ou plus tard encore dans la philosophie de Platon.

\section{La réponse de Platon à la crise dionysiaque}

21 Cassirer prend pour objet de réflexion la manière dont le disciple de Socrate affronta la question de la crise provoquée par la propagation en Grèce des nouveaux cultes dionysiens :

Toutes les conceptions homériques furent balayées au cours d'une profonde crise culturelle et religieuse. La sérénité et la simplicité des Olympiens disparurent alors apparemment complètement. Zeus le dieu du Ciel et Apollon le dieu du soleil cessèrent de pouvoir repousser les forces démoniaques apparues avec le culte de Dionysos. Ce dernier n'avait pas sa place chez Homère [...]. La religion grecque devint dès lors le théâtre d'un combat entre deux forces adverses. On en trouve l'expression désormais classique dans Les Bacchantes d'Euripide. Une simple lecture de ce dernier suffit pour se rendre compte de la violence et de la force qui furent celles à l'époque de ce nouveau culte religieux (Cassirer 1993, p. 64).

Les mutations engendrées par les nouveaux cultes dionysiaques, repris et adaptés à la tradition religieuse et culturelle grecque dans le cadre de l'orphisme, instituèrent des rites violents :

L'orphisme transforma en un système tout un ensemble originellement constitué de rites primitifs des plus sauvages et des plus frustes. Sa théologie élabora la légende de Dionysos Zagreus, décrit comme le fils de Zeus et de Sémélé. Celle-ci sommait les titans de le tuer enfant. Il parvenait à leur échapper par des métamorphoses successives avant de prendre la forme d'un taureau [...] son corps finissait par être déchiqueté en morceaux que ses ennemis dévoraient (p. 65).

Devant la violence cyclique à laquelle le retour farouche du rite et du mythe condamnait la société, Platon manifestera dans toute son œuvre le souci de sortir du mythique par le logos philosophique. Il construisit par la suite une théorie éthique et politique de l'État. Sa théorie a été fortement marquée par la crainte des débordements du mythe qui pourraient se loger jusque dans la poésie :

Platon deviendra l'ennemi déclaré du mythe [...] Il n'y a qu'une alternative : nous devons choisir entre une conception éthique [ou] mythique de l'État. [...] Tant que nous continuerons à parler de guerre dans le ciel, [...] de batailles livrées par des titans ainsi que les innombrables querelles opposant les dieux et les héros [...] nous ne trouverons jamais aucun ordre, aucune harmonie, ni aucune unité dans notre 
propre monde. (La République, Livre 2, 377 sq) [...] l'une des tâches principales que se donnera Platon consistera à tout faire pour briser le pouvoir de cet «éternel hier » (pp. 104-105).

Dans le chapitre V, «Logos et mythos dans la philosophie grecque » (pp. 79-88), Cassirer souligne les forces mises dans la philosophie grecque pour lutter contre les conceptions mythiques, de l'histoire, de la physique, de la philosophie et même de la théologie (p. 82). Il insiste sur le combat pour s'affranchir de la pensée mythique, pour instituer la séparation entre mythos et logos. Le mérite de Socrate était d'instaurer une révolution éthique :

Pour dépasser le pouvoir du mythe, il fallait trouver et développer le nouveau pouvoir positif de la «connaissance de soi ». Il fallait apprendre à envisager le tout de la nature humaine dans une perspective éthique et non plus mythique. Selon Socrate, le mythe pouvait apprendre beaucoup de choses aux hommes, mais il ne possédait aucune réponse à la seule chose qui importait, celle du bien et du mal. Seul le Logos socratique, seule la méthode d'introspection introduite par Socrate en personne pouvait apporter une réponse à la solution de ce problème fondamental et essentiel (p. 88).

Cassirer, dans le Chapitre VI, consacré à la République de Platon, montrera le brillant disciple attaché à construire une approche rationnelle de l'État, autant système politique que théorie de l'éducation qui est autant politeïa que païdeia (p.92). Il expose la philosophie de Platon comme effort méthodique, à la fois éthique, politique et éducatif pour surmonter le mythe et protéger ainsi l'esprit comme la cité contre l'intrusion des forces obscures du mythe. Pour comprendre ces transformations, il peut être intéressant de confronter les réponses des anthropologues (Durkheim; Mauss, 2001; Girard, 2004 ; Tarot, 2008 \& 1999) à celles qu'a pu ou que pourrait apporter la philosophie, grâce à la pensée de Cassirer. La première tournée vers une genèse de la culture comme anthropologie du sacré et du symbolique, l'autre tournée vers la théorie de la culture par une philosophie critique des formes symboliques.

\section{Conclusions}

Après avoir réfléchi à la place des catégories du mythico-magique, après avoir esquissé un repérage des fonctions du mythique, Cassirer a signalé les forces d'engendrement de toutes les formes symboliques jusqu'aux plus élevées, la rationalité scientifique, l'éthique, dans le mythe. À la fin de son œuvre, Cassirer a attiré l'attention sur le retour catastrophique du mythique, engendrant des formes confuses et violentes de la culture, à travers l'instrumentalisation majeure du bouc émissaire. Dans Le mythe de l'État, publié pour la première fois en 1946, l'auteur démonte la manipulation mythique et son instrumentalisation politique dans la culture nationale-socialiste de l'Allemagne hitlérienne. Cassirer permet de comprendre le nazisme comme phénomène culturel total qui renoue avec le mythico-magique et fait refleurir à contretemps les catégories caractéristiques telles que la substantialisation, le bouc émissaire, l'indifférenciation, ou encore la performativité magique dans la manipulation du langage. Il s'est attaché pendant plus de vingt ans à réfléchir aux caractéristiques du mythe dans ses fonctions, à l'aube des civilisations, de genèse de la rationalité et de la culture, puis de latence plus ou moins créative, enfin son ambivalence et ses dysfonctions catastrophiques et en a su dégager quelques outils pour le circonscrire. 

mythique peut détruire une culture, surtout quand elle offre dans les situations de crises culturelles l'hybridation indifférenciée des savoirs ou quand, par ce qu'il a fortement désigné comme "transvaluation", les valeurs migrent illégitimement d'un système symbolique à l'autre. Ainsi, après Cassirer et sur ses pistes, tandis qu'une réflexion critique sur la culture s'avère possible et nécessaire, n'est-il pas urgent aujourd'hui de comprendre la destructivité du mythe du marché ou les mythes des États fondamentalistes? Après la crise des années 1940, avec la défaite du nazisme, à la Libération, les États européens avaient voulu engager une mutation éthico-politique. Toutefois, la crise actuelle du marché globalisé, avec le creusement abyssal des inégalités, avec la montée des risques écologiques, avec les migrations économiques, politiques et bientôt climatiques de masse, le retour des mécanismes d'un sacré archaïque et les processus enchevêtrés de boucs émissaires, demande de pousser l'analyse réflexive plus loin. Actuellement, on a l'impression que cette crise a bien dévoyé le rêve de l'institution d'une Europe généreuse ou du partage universalisé d'une mondialisation heureuse.

La réflexion de Cassirer dessine la tâche à accomplir d'une analyse de la «transvaluation de toutes les valeurs", de la confusion des ordres tapie au cœur de la novlangue mondialisée du management néolibéral, elle esquisse enfin la manière de décrypter les traces d'un nouveau sursaut politico-éthique. Sans partager les illusions naïves des Lumières triomphantes, Cassirer montre la voie d'une confiance persistante et efficiente dans les pouvoirs de la rationalité. Rationalité différenciée selon les spécificités des domaines symboliques, avertie sur les dangers de l'indifférenciation qui toujours ramène les périls du mythico-magique avec son cortège de boucs émissaires :

La philosophie est capable de rendre de grands services. Elle peut, en effet, aider à faire comprendre qui est l'adversaire que l'on combat. [...] il faut que l'on se mette à étudier soigneusement l'origine, la structure et la technique des mythes politiques. Il faut que nous apprenions à regarder l'adversaire en face, afin de savoir comment le combattre (Cassirer, 1993, p. 400).

\section{BIBLIOGRAPHIE}

Cassirer, E. (1962). Leibniz' System in seinen wissenschaftlichen Grundlagen (1902). Hildesheim : G. Olms.

Cassirer, E. (1966). La philosophie des Lumières (1932). Paris : Fayard.

Cassirer, E. (1972)1. La philosophie des formes symboliques 2 : La pensée mythique (1925). Paris :

Éditions de Minuit.

Cassirer, E. \& Heidegger, M. (1972). Débat sur le Kantisme et la philosophie (Davos, mars 1929) et autres textes de 1929-1931. Paris : Beauchesne.

Cassirer, E. (1993). Le mythe de l'État (1946). Paris : Gallimard. 
Durkheim, É. (1898). L'individualisme et les intellectuels (initialement publié dans la Revue bleue, 4 e série). Mis en ligne par l'UQAC, coll. « Les classiques des sciences sociales ». DOI : http:// dx.doi.org/doi:10.1522/cla.due.ind

Durkheim, É. (1975). Religion, morale, anomie : Textes II. Paris : Les Éditions de Minuit.

Durkheim, É. (1975). Lettre à Célestin Bouglé (1898), dans Textes II (p. 423). Paris : Les Éditions de Minuit.

Durkheim, É. (1985). Les formes élémentaires de la vie religieuse (1912). Paris : PUF.

Durkheim, É. (1992). L'éducation morale (3éd.). Paris : PUF.

Durkheim, É. (1998). De la division du travail social (1893). Paris : PUF/Quadrige.

Flood, A. (éd.) (2008). Ernst Cassirer. The Myth of the State. Repéré à http:// www.anthonyflood.com/cassirermythstate.htm

Girard, R. (1972). La violence et le sacré. Paris : Grasset.

Girard, R. (1978). Des choses cachées depuis la fondation du monde. Paris : Grasset.

Girard, R. (1982). Le bouc émissaire. Paris : Grasset.

Girard, R. (2004). Les origines de la culture. Paris : Desclée de Brower.

Mauss, M. (2001). Sociologie et anthropologie. Paris : PUF.

Recki, B. (2013). Cassirer. Stuttgart : Reclam.

Tarot, C. (1999). De Durkheim à Mauss. L'invention du symbolique; sociologie et science des religions.

Paris : Éditions La Découverte/MAUSS.

Tarot, C. (2008). Le symbolique et le sacré, Théories de la religion. Paris : Éditions La Découverte/ MAUSS.

\section{NOTES}

1. Sont parues la même année, chez le même éditeur, les traductions des deux autres volumes : Le langage (1923) et La phénoménologie de la connaissance (1929).

\section{RÉSUMÉS}

Pour Ernst Cassirer, la confusion qui marque la crise culturelle est toujours liée à la résurgence d'une pensée mythique. Notre étude se propose, pour comprendre les confusions actuelles, de reprendre les réflexions philosophiques de Cassirer, et leur inspiration anthropologique, notamment autour des notions d'indifférenciation et de «bouc émissaire » comme catégories du mythico-magique. Une place privilégiée est accordée ici à cet auteur dont la philosophie des formes symboliques offre des ressources analytiques et critiques inestimables et jusqu'alors peu reconnues. 
For Ernst Cassirer, the confusion marking a cultural crisis is always linked to the resurgence of mythical thought. In order to understand the current confusion, our study proposes to refer at the philosophical reflections of Cassirer and their anthropological inspiration, in particular the notions of indifferentiation and "scapegoat" as categories of the mythico-magic. A privileged place is granted to this author whose philosophy of symbolic forms offers invaluable analytical and critical resources hitherto little recognized.

INDEX

Keywords : indifferenciation, scapegoat, mythical-magic, symbolic forms, ethical-politic Mots-clés : indifférenciation, bouc émissaire, mythico-magique, formes symboliques, éthicopolitique

\section{AUTEURS}

\section{LEONORE BAZINEK}

Chercheuse associée au laboratoire ERIAC, Université de Rouen

\section{MARIE-LOUISE MARTINEZ}

Professeure des Universités, Laboratoire CIVIIC, Université de Rouen 